\title{
Driving forces for texture transformation in thin Ag films
}

Elizabeth A. Ellis ${ }^{1, *}$, Markus Chmielus ${ }^{1,2, *}$, Ming-Tzer Lin ${ }^{1,3}$, Howie Joress ${ }^{1,4}$, Kyle Visser ${ }^{1}$, Arthur Woll ${ }^{4}$, Richard P. Vinci ${ }^{5}$, Walter L. Brown ${ }^{5}$, and Shefford P. Baker ${ }^{1, \ddagger}$

1. Cornell University, Department of Materials Science and Engineering, Bard Hall, Ithaca, NY 14853 USA

2. University of Pittsburgh, Department of Mechanical Engineering \& Materials Science, Benedum Hall, Pittsburgh, PA 15261 USA

3 National Chung Hsing University, Graduate Institute of Precision Engineering, 402, Taichung, Taiwan, ROC

4 Cornell University, Cornell High Energy Synchrotron Source, Wilson Lab, Ithaca, NY 14853 USA

5. Lehigh University, Department of Materials Science and Engineering, Whitaker Laboratory, 5 East Packer Ave, Bethlehem, PA 18015 USA

* These two authors contributed equally to this work

\$ Corresponding author

\section{ABSTRACT}

The well-known thickness-dependent (111)-to-(100) texture transformation in thin FCC films is usually attributed to a competition between interface and strain energies. In this model, thin films retain their (111) texture due to the lower energy of the (111) interface, while thick films transform to (100) due to the lower stiffness and thus strain energy of a (100) film. However, recent work has called this model into question, suggesting that neither the stress nor the interface energy play a dominant role in texture transformation. We investigated the driving forces involved in this transformation by using a bulge test apparatus to induce different stresses in thin Ag films under identical annealing conditions. In situ synchrotron XRD measurements show the change in texture during annealing, and reveal that applied stresses have no effect on the transformation. Stress analysis shows that differences in driving forces for texture transformation due to applied bulge pressure were significant $\left(\approx 200 \mathrm{~kJ} / \mathrm{m}^{3}\right)$, suggesting that a different, much larger driving force must be responsible. Reduction in defect energy has been proposed as an alternative. However, vacancy and dislocation densities must be exceptionally high to significantly exceed the strain energy and do not provide obvious orientation selection 
mechanisms. Nanotwins in reported densities are shown to provide greater driving force $(\approx 1000$ $\mathrm{kJ} / \mathrm{m}^{3}$ ) and may account for orientation selection. The large difference between the calculated strain and defect energies and the driving force for grain growth $\left(21,100 \mathrm{~kJ} / \mathrm{m}^{3}\right)$ casts doubt on the applicability of a simple thermodynamic model of texture transformation. 


\section{INTRODUCTION}

A thickness-dependent (111)-to-(100) texture transition is well known in thin FCC metal films. Fine-grained structures having (111) fiber texture (grains with (111) planes parallel to the film plane and random orientations in the plane of the film) are produced by many deposition processes, regardless of film thickness [1]. During subsequent annealing, the behavior depends on the film thickness. In films that are sufficiently thin, the (111) grains grow normally and the (111) texture is retained. In films that are sufficiently thick, (100)-oriented grains grow at the expense of (111) grains, transforming the film to a (100) fiber texture by what has been described as an abnormal grain growth process [2,3]. Since the orientation distribution determines properties, there has been much interest in this texture transformation [2-17].

The (111)-to-(100) texture transformation has been attributed [2-4] to a competition between strain energy and interface energy. In FCC materials, the (111) orientation has the lowest interface energy, while the (100) orientation has the lowest biaxial modulus. Thus, the interface energy per unit volume decreases as the inverse of the film thickness, while, at a given strain, the strain energy per unit volume is constant with film thickness. This model therefore predicts that a critical thickness exists, below which a film should adopt (111) fiber texture to minimize interface energy, and above which it should adopt a (100) fiber texture to minimize strain energy. This interface/strain energy model is widely cited $(e . g .[18,19])$ and appears to be the reigning explanation for this phenomenon.

However, recent studies have questioned the role of both stresses and interface energies in this texture transformation. Where stresses are known with certainty, they have been shown to be insufficient to produce the texture transition [10,11, 15-17]. Indeed, even films removed from their substrates and thus nominally stress-free have been observed to transform $[10,11]$. Furthermore, the transitions seem to occur in films of similar thickness regardless of the interface

conditions $[10,17]$. Thus, it is evident that additional driving forces must be in play. Elimination of microstructural defects (e.g. point defects, dislocations, and planar defects) has also been 
proposed as a driving force for the texture transformation $[15,17]$. However, very little work has been done to identify, separate, and quantify the different driving forces associated with the texture transformation. In only a handful of studies [15-17] have the stresses actually been measured, and even more rarely has the reduction in defect energy been considered [15, 17]. One complication is that films of different thicknesses have not only different stresses [16], but different microstructures $[10,11,15]$, and thus possibly different combinations of driving forces that conspire to direct the texture transformation.

We have conducted experiments designed to impose different strain energies as driving forces for the transformation, independent from interface and defect energies, so that the results could be compared with energies arising from elimination of different defect types (point, line, and planar). To do this, we used a bulge test apparatus to impose widely different stresses on thin $\mathrm{Ag}$ films during annealing and tracked the texture transformation in-situ using synchrotron $\mathrm{x}$-ray diffraction. All films had the same initial structure and thickness. Remarkably, the imposed strain energies had no effect on the kinetics of the texture transformation, indicating that much larger driving forces must be at work. Furthermore, the previously neglected strains that arise due to reduction in excess free volume during grain growth were found to be significant during the transformation. Calculations show that the strain energy contribution to the driving force for transformation was successfully varied over a range of about $200 \mathrm{~kJ} / \mathrm{m}^{3}$, suggesting that the actual driving force must be much larger. If reduction in defect energy is indeed that driving force, then a minimum defect density with a strong orientation dependence in the as-deposited films is necessary. We show that extremely high densities of vacancies or dislocations would be required, but don't provide an obvious orientation selection mechanism. However, nanotwins in experimentally reported densities can provide a driving force relative to the opposing strain energy and may also provide an orientation selection mechanism. All of the driving forces considered were much smaller than the driving force for grain growth $\left(\approx 21,100 \mathrm{~kJ} / \mathrm{m}^{3}\right)$ suggesting that kinetics must also play a role. 


\section{EXPERIMENTAL}

Substrates were fabricated by etching through the thickness from the backside of $\mathrm{Si}_{3} \mathrm{~N}_{4}$-coated, (001)-oriented, $20 \mathrm{~mm} \mathrm{!20} \mathrm{mm} \mathrm{Si} \mathrm{dies} \mathrm{to} \mathrm{create} \mathrm{either} \mathrm{a} 2 \mathrm{~mm} ! \llbracket 2 \mathrm{~mm}$ or a $3 \mathrm{~mm} ! \llbracket 2 \mathrm{~mm}$ rectangular nitride membrane in the center of each die (Figure 1a and b, and Table 1). The $\mathrm{Si}_{3} \mathrm{~N}_{4}$ layer was $210 \mathrm{~nm}$ thick for all dies.

A titanium adhesion layer followed by a silver film were then deposited onto the nitride on the front side of each die using e-beam evaporation at room temperature. Ti was deposited from $99.995 \%$ pure pellets at a rate of $0.5 \mathrm{~nm} / \mathrm{s}$ to a thickness of $40 \mathrm{~nm}$. Silver films were deposited on top of the Ti adhesion layer from $99.99 \%$ pure pellets at a rate of $4.0 \mathrm{~nm} / \mathrm{s}$ to a nominal thickness of $1200 \mathrm{~nm}$. Both the silver and titanium layers were evaporated out of molybdenum crucibles. The chamber base pressure was between $8.9 \times 10^{-7}$ and $1.9 \times 10^{-6}$ Torr $\left(1.2-2.5 ! 10^{-4} \mathrm{~Pa}\right)$ for all depositions. Film thicknesses were measured after deposition using contact profilometry (Table 1).

Each sample was then mounted onto a bulge tester that consists of a chamber cut into a brass block with a port and a gas inlet tube (Fig. 1b). The sample was clamped over the port using a metal retaining ring that pushed the die against a Viton O-ring, providing a gas-tight seal. The chamber could then be pressurized with helium, causing the $\mathrm{Si}_{3} \mathrm{~N}_{4} / \mathrm{Ti} / \mathrm{Ag}$ membrane in the sample to bulge out as shown in Fig. 1c, inducing a net tensile stress in the membrane. The bulge tester was attached to an OFHC copper base block (Fig. 1b) whose temperature was controlled by flowing water from a thermostat-controlled heater through a channel in the base block. The temperature was measured using a thermocouple attached to the upper portion of the bulge tester. The measured temperature could be maintained within about $2{ }^{\circ} \mathrm{C}$ at a nominal annealing temperature of $70{ }^{\circ} \mathrm{C}$.

Texture volume fractions were determined in-situ during annealing using synchrotron x-ray diffraction (XRD). The bulge tester was mounted in a 6 -circle $\kappa$ goniometer [20] at the G2 beamline of the Cornell High Energy Synchrotron Source (CHESS). The X-ray beam from a Be crystal monochromator had $\mathrm{LaB}_{6}$ (NIST SRM 660) calibrated wavelength of $1.2952 \AA$ and a 
bandwidth of approximately $0.1 \%$. The incident beam, with dimensions of 0.2 ! $2.0 \mathrm{~mm}$ defined by a set of slits, was aligned such that it was centered on the bulge with the long axis of the rectangular beam parallel to the long axis of the bulge. The diffraction geometry is shown in Figure 2. Diffracted beam intensities were measured using a 2-D detector with a point-to-point resolution of 0.0113 "that spanned an angular range of 2.2 " in 2 ! \#and 5.5 " in the horizontal plane at $2 \theta=0$ ". Use of the 2-D detector was necessary because the transformed films were expected to have very large grain sizes [11]. By capturing a portion of the diffraction cone $( \pm \psi)$, the chances of capturing peaks from large grains with slight misorientation were increased. Rocking curves were generated by rotating the sample about $\omega$, an axis perpendicular to the incident beam and parallel to the sample surface ( $\omega$ geometry). The sample height was aligned by setting the beam at $! \models 0^{\circ}$ to correspond to the top of the sample retainer and then offsetting by the thickness of the retainer. The maximum bulge height was about $50 \mu \mathrm{m}$ and was neglected during diffraction experiments on bulged films, introducing a small height error. To minimize air scattering, a He-filled flight path was installed between sample and detector. $\mathrm{A} \mathrm{N}_{2}$ ion chamber upstream from the sample provided a continuous measurement of the incident beam intensity.

To study the effect of stress on the texture transformation, three different samples were tested, each at different applied bulge pressures, and therefore film stresses (Table 1). The highest pressure level was selected to provide as much bulge displacement as possible without risk of bursting the membrane during the experiment. One sample was tested with zero applied pressure, and the third was tested at an intermediate pressure. For all three samples, the procedure was the same: First, the bulge pressure was set. Then, a $\theta-2 \theta$ scan covering a range of $30^{\circ} \leq 2 \theta \leq 80^{\circ}$ ( (step size $0.0667^{\circ}$ ) was performed at room temperature. Next, the water flow to the heater block was started. The sample temperature reached $67^{\circ} \mathrm{C}$ within 3 minutes, and stabilized at a final temperature between 67 and $70{ }^{\circ} \mathrm{C}$ (Table 1) within another 5 minutes. A set of four scans of the (222) and (400) silver diffraction peaks, including $2 \theta$ scans $\left( \pm 1^{\circ}\right.$ around the peak center, step size $\left.0.05^{\circ}\right)$ as well as $\omega$ rocking curve scans $\left( \pm 10^{\circ}\right.$ around the film normal, step size $1^{\circ}$ ) of both peaks, was conducted. The photon count was recorded at each pixel in the 2D 
detector for $0.5 \mathrm{~s}$ at each point and a full set of four scans lasted on the order of 4 minutes. These measurements began when heating was started and the full set of four scans was repeated continuously until the end of the experiment. In all three samples, the (222) peak intensity declined and the (400) peak intensity increased during annealing. The XRD scan sequence was terminated when the (400) peak intensity stopped increasing. A final $2 \theta$ scan from 30 to $80^{\circ}$ was conducted before the bulge pressure was released and the bulge tester and sample were cooled to room temperature.

Following the XRD measurements, the bulge tester was removed from the diffractometer and the stress-strain behavior of the annealed film was determined using a bulge test to obtain an accurate measurement of the stress in the film during the XRD experiments. The bulge height, $h$, was measured as a function of pressure, $p$, using a simple Fabry-Perot optical interferometer with the bulge as the fully reflective surface and an optically flat half-silvered mirror as a reference surface. A CCD camera was used to record interference fringe motion as the pressure was increased. The bulge height was obtained by counting the number of fringes that passed a given point. A laser light source with a $532 \mathrm{~nm}$ wavelength gave a height resolution of $266 \mathrm{~nm}$.

\section{RESULTS}

The texture evolution was determined from the x-ray data and the stresses were determined from the post-annealing bulge test pressure-height data.

\subsection{Texture evolution}

The only peaks seen during $2 \theta$ scans performed both before and after transformation were those associated with (111) and (100) texture components. All films had strong (111) texture before annealing, and strong (100) texture after annealing. Figure 3 shows detector images of the diffracted (222) intensity before, and the (400) intensity after annealing, respectively. The (222) intensity forms a smooth ring segment, consistent with the expected fine (111) grain size in the as-deposited films. The (400) intensity occurs in a few intense spots, consistent with the presence 
of only a few large (100) grains produced by abnormal grain growth during annealing. For the $\omega$ rocking curve scans, the diffracted intensity associated with each measurement was taken to be the total intensity collected by the detector (after background subtraction) at that point - i.e. the integrated intensity over the small segment in $\psi$ and $2 \theta$ captured by the detector (e.g. the total intensity from detector images such as those in Fig. 3), which included only the peak of interest. Figures $4 \mathrm{a}$ and $\mathrm{b}$ show the (222) rocking curve before and the (400) rocking curve after annealing for the sample tested at $0 \mathrm{kPa}$ applied pressure. Again, the smoothness of the (222) rocking curve is consistent with the expected fine grain size, while the jagged appearance of the (400) rocking curve arises because of the very large (100) grains in the annealed films. (Note that, since the intensity at each point in the rocking curve is the total detector intensity at that point (e.g. Fig. 3b), the peaks in the rocking curve don't necessarily correspond to individual grains.) The evolution of the (222) and (400) rocking curve peaks as a function of time during annealing for the sample tested at zero applied bulge pressure is presented in Figures $4 \mathrm{c}$ and $\mathrm{d}$, which show that the texture transformation occurred smoothly over a period of about 4 hours.

Since only peaks corresponding to the (100) and (111) texture components were found, the volume fraction of the 100 texture component, $f_{100}$, can be estimated by comparing the normalized integrated intensity of the (400) peak with the combined normalized integrated intensities of the (400) and the (222) peaks:

$$
f_{100}=\frac{I_{400}}{I_{400}+I_{222}}
$$

$I_{222}$ is taken to be the area under a Gaussian fitted to each (222) rocking curve, and, because of the irregular shape of the (400) rocking curves, $I_{400}$ was calculated by summing the intensity over the points in each rocking curve.

To account for slight variations in x-ray flux, all count measurements were normalized to the incoming flux as measured by the ion detector upstream of the sample. Corrections were also made to account for changes in sample diffraction volume, Lorentz factor, and scattering factor 
(structure factor is the same for both peaks) as well as for the solid angle covered by the detector at each $2 \theta$ value for each peak. Due to the imperfect alignment of the incident beam to the peak of the bulge, the sample normal was not parallel to the goniometer normal. To account for this, the $\omega$ value was shifted by moving the center of the rocking curve for the first (222) peak to zero and the offset applied to all other data taken for that sample.

The texture transformation is quantified by plotting $f_{100} v s$. time as shown in Figure 5 . As is evident from the sample tested at an applied bulge pressure of $0 \mathrm{kPa}$, the texture transformation follows a typical "s-shaped" curve typical of nucleation and growth processes and seen previously in texture transformations $[15,17]$. The first sample was tested approximately 27 hours after being removed from the deposition chamber and was untransformed (Fig's 3 and 4) at the start of the test. However, the second sample $(p=5 \mathrm{kPa})$ was tested approximately 36 hours after deposition and had an initial value of $f_{100}=0.44$, while the third sample $(p=13 \mathrm{kPa})$ was tested 41 hours after deposition and had an initial value of $f_{100}=0.45$. It has been reported that small amounts of impurities dramatically inhibit the texture transformation in Ag films [17]. These results show that if sufficiently pure films are produced, samples transform readily even at room temperature on the scale of days. The transformation curves can be compared if the transformation data are shifted to account for the extent of transformation that occurred before $\mathrm{x}$ ray measurements began. As evident in Fig. 5d, all three samples show virtually indistinguishable transformation kinetics.

\subsection{Stress calculations}

Ideally, one could determine the stresses using the strains calculated from the plane spacing measured using x-rays. However, the bulge test tester did not have sufficiently precise temperature control for this, and the geometry and time precluded measurements of the stresses in the few large (100) grains from which diffraction peaks were captured. Instead, we used the bulge test to determine the stresses. The bulged films consisted of $\mathrm{a} \approx 210 \mathrm{~nm} \mathrm{Si}_{3} \mathrm{~N}_{4}$ layer, $\mathrm{a} \approx 40$ $\mathrm{nm}$ Ti layer and $\mathrm{a} \approx 1200 \mathrm{~nm} \mathrm{Ag}$ layer. The $\mathrm{Si}_{3} \mathrm{~N}_{4}$ layer, as prepared, had a stress at room 
temperature of $424 \mathrm{MPa}$ [21]. We ignore any stress in the thin Ti layer, and seek an estimate of the stresses in the Ag layers under pressure and temperature at the beginning of the in-situ annealing experiment (and thus the strain energy available to drive the texture transformation) using a bilayer analysis.

The length-to-width aspect ratios of the rectangular bulged films (Fig. 1a and Table 1) were all greater than 4 allowing us to use a plane strain bulge test analysis [22, 23]. We modify this analysis for the case of a bilayer membrane comprised of layer 1 with thickness $t_{1}$ residual stress $\sigma_{1,0}$, and plane strain modulus $S_{1}$, and layer 2 having thickness $t_{2}$, residual stress $\sigma_{2,0}$, and plane strain modulus $S_{2}$. The total force per unit width along the edges of the bulge membrane, $F_{\mathrm{T}}$ is just the sum of the edge forces in the two layers so that

$$
F_{T}=F_{1}+F_{2}=\sigma_{T} t_{T}=\sigma_{1} t_{1}+\sigma_{2} t_{2} .
$$

Thus, the plane strain pressure-height relation presented by Vlassak and Nix [22] and Xiang and Vlassak [23] can be modified for the biaxial bilayer case, resulting in

$$
p=\frac{2}{a^{2}}\left(\sigma_{1,0} t_{1}+\sigma_{2,0} t_{2}\right) h+\frac{4}{3 a^{4}}\left(S_{1} t_{1}+S_{2} t_{2}\right) h^{3} .
$$

In our case, layer 1 is $\mathrm{Si}_{3} \mathrm{~N}_{4}$ for which we use the subscript $\mathrm{SiNx}$ and layer 2 is $\mathrm{Ag}$ (subscript $\mathrm{Ag}$ ). The $\mathrm{Si}_{3} \mathrm{~N}_{4}$ layer is amorphous and therefore isotropic, so its plane strain modulus can be found using $S_{\mathrm{SiNx}}=E_{S i N x} /\left(1-v_{S i N x}^{2}\right)$ with $E_{S i N x}=150 \mathrm{GPa}$ and $v_{\mathrm{SiNx}}=0.24$ [21] giving $S_{\mathrm{SiNx}}=159$ $\mathrm{GPa}$. The Ag layer is anisotropic. Using tensor elastic constants, we find

$$
S_{A g}^{100}=\frac{3 C_{11}^{2}-4 C_{12}^{2}+C_{11}\left(C_{12}+C_{44}\right)+C_{11}\left(C_{11}-C_{12}-C_{44}\right) \cos (4 \phi)}{4 C_{11}}
$$

and

$$
S_{A g}^{111}=\frac{3 C_{11}^{2}+3 C_{11} C_{12}-6 C_{12}^{2}+22 C_{11} C_{44}+16 C_{44}^{2}}{7 C_{11}+11 C_{12}+22 C_{44}}
$$


where $\phi$ is the azimuthal angle relative to the [100] direction. $S_{A g}^{111}$ is not a function of $\phi$ and can be evaluated explicitly. Since $S_{A g}^{100}$ varies with azimuthal angle, the average over $\phi$ (roughly appropriate for a fiber texture) may be calculated instead. Using published values for single crystal elastic constants [24], this gives plane strain moduli of $S_{A g}^{100}=69$ and $S_{A g}^{111}=112 \mathrm{GPa}$.

In principle, one could perform a bulge test of the as-deposited films to determine both the stress in the as-deposited state and the stress under pressure. However, the membrane regions were wrinkled in the as-deposited samples, indicating a net compressive stress at room temperature that made quantitative bulge test measurements impossible $[23,25]$. However, the membranes were flat at room temperature after annealing, indicating a net tensile stress. Thus, we conducted bulge tests at room temperature in the annealed films to find the residual stress, and estimated the stress in the Ag layer at the start and end of the transformation by accounting for the changes in stress due to temperature, the texture transformation, grain growth, and the bulge pressure.

This procedure is illustrated in Figure 6. Our approach is to go step by step to describe the changes in stress that must occur during the experimental procedures (solid lines, left to right in Fig. 6), then, knowing the stresses in the final annealed films from the ex-situ bulge tests, we calculate the actual stresses in the films by going through the steps in reverse order (dotted lines, right to left in Fig. 6). Note that, since the stress values depicted in Fig. 6 reflect only the behavior of the Ag layer, the 'Ag' subscript is omitted in this figure for clarity. In this work, the $\mathrm{Si}_{3} \mathrm{~N}_{4}$ layer is assumed to behave elastically throughout the experiment and the contribution of the Ti layer is assumed to be negligible.

We begin with a thought experiment to describe the stresses and their origins during the experimental procedure (solid lines in Fig. 6). The Ag layer starts out in the (111) orientation at room temperature. For the moment, we neglect the fact that the bilayers containing the asdeposited films were wrinkled and treat them as if they could somehow be artificially held flat (i.e. not buckled), so that the Ag film there would have the same residual compressive stress, 
$\sigma_{A g, 0, R T}^{111}$, that it must have in regions where it is directly over the Si substrate. This will allow us to find the correct strain state when the film is bulged.

When the temperature is raised (and the bilayer remains flat!), a thermal strain is imparted to each layer, which, if the temperature dependence of the thermal expansion coefficients can be neglected, is given by

$$
\Delta \varepsilon_{t h}=\left(\alpha_{S i}-\alpha_{f i l m}\right)\left(T_{f}-T_{i}\right),
$$

where $\alpha_{\mathrm{Si}}$ and $\alpha_{\text {film }}$ are the thermal expansion coefficients of the Si substrate and the $\mathrm{Si}_{3} \mathrm{~N}_{4}$ or $\mathrm{Ag}$ layers, respectively, and $T_{\mathrm{f}}$ and $T_{\mathrm{i}}$ the final and initial temperatures. This leads to a stress in the (111) Ag layer of $\sigma_{A g, 0,70}^{111}$. When the bulge pressure is applied, an additional strain given by

$$
\Delta \varepsilon_{b}=\frac{2 h^{2}}{3 a^{2}},
$$

is added to both layers, and the stress in the (111) Ag layer, now at the test temperature (subscript ' 70 ') and pressure (subscript ' $p$ '), is $\sigma_{A g, p, 70}^{111}$. When the film then undergoes the texture transformation, two things happen that change the stress and strain state. First the grains grow, eliminating excess free volume from the film and inducing an additional tensile strain given by

$$
\Delta \varepsilon_{g g}=\delta\left(\begin{array}{ll}
\frac{1}{\mathrm{~d}_{i}} & \frac{1}{\mathrm{~d}_{f}}
\end{array}\right),
$$

where $\delta$ is the grain boundary width and $d_{\mathrm{i}}$ and $d_{\mathrm{f}}$ are the initial and final grain sizes [26]. Second, the new grains that grow have the (100) orientation. This does not change the strain directly, but the lower modulus means that the Ag layer supports less stress at a given strain, causing more of the total load to be transferred to the $\mathrm{Si}_{3} \mathrm{~N}_{4}$ layer. These processes occur simultaneously, but we can think of either letting the grains grow first, leading to $\sigma_{A g, p, 70, g g}^{111}$, and then changing the orientation, or we can change the orientation first, leading to $\sigma_{A g, p, 70}^{100}$, and then letting the grains grow. Either way, the layer ends up at $\sigma_{A g, p, 70, g g}^{100}$, the stress state of a pressurized, fully transformed film. Next, the bulge pressure is reduced to zero, inducing a change in bulge strain (Eq. 7) leading to $\sigma_{A g, 0,70, g g}^{100}$, as shown in Fig. 6. Finally, the sample is 
cooled to room temperature, inducing a tensile thermal strain (Eq. 6), and leading to a final stress of $\sigma_{A g, 0, R T, g g}^{100}$.

Since the real films were observed to be flat at the end of the actual experimental process, we used the bulge test (Eq. 3) to directly measure the residual stress $\sigma_{A g, 0, R T, g g}^{100}$ in the transformed Ag films at room temperature. We could then calculate the values we want, $\sigma_{A g, p, 70}^{111}$ and $\sigma_{A g, p, 70, g g}^{100}$ (points marked with stars in Fig. 6), by simply working backwards, from right to left in Fig. 6, as indicated by the dashed lines, assuming that: (1) the films remain flat until the bulge pressure is applied, and (2) no plasticity occurs.

First, we fit Eq. 3 to bulge test data (not shown). We use the values for the plane strain moduli and the residual stress in the nitride described above (Table 1). The residual stress in the Ag layer, $\sigma_{A g, 0}=\sigma_{A g, 0, R T, g g}^{100}$, is the sole variable and is found to be $100 \pm 5 \mathrm{MPa}$. Next, using Hooke's law and the equal-biaxial moduli $Y_{A g}^{100}=75 \mathrm{GPa}[17]$ and $Y_{\mathrm{SiNx}}=E_{S i N x} /\left(1-v_{S i N x}\right)=197$ $\mathrm{GPa}$, we find the residual strains in the $\mathrm{Ag}$ and nitride layers, $\varepsilon_{A g, 0, R T, g g}^{100}$, and $\varepsilon_{\mathrm{SiNx}, 0, \mathrm{RT}}$, respectively, from the residual stresses. We then imagine heating the samples to the test temperature. The residual strains then become

$$
\varepsilon_{S i N x, 0,70}=\varepsilon_{S i N x, 0, R T}+\left(\alpha_{S i}-\alpha_{S i N x}\right)\left(T_{70}-T_{R T}\right)
$$

And

$$
\varepsilon_{A g, 0,70, g g}^{100}=\varepsilon_{A g, 0, R T, g g}^{100}+\left(\alpha_{S i}-\alpha_{A g}\right)\left(T_{70}-T_{R T}\right)
$$

Using values for $\alpha_{S i}, \alpha_{S i N x}$ and $\alpha_{A g}$ of $2.6 \times 10^{-6} \mathrm{~K}^{-1}[27], 3.3 \times 10^{-6} \mathrm{~K}^{-1}[28]$, and $16.5 \times 10^{-6} \mathrm{~K}^{-1}$ [29], we find that the thermal strains for this heating process are $-3.43 \times 10^{-4}$ and $-8.28 \times 10^{-4}$ in the nitride and silver layers respectively (Table 1). We again apply Hooke's law using the biaxial moduli as described above to find the residual stresses $\sigma_{\mathrm{SiN} x, 0,70}=Y_{\mathrm{SiNx}} \varepsilon_{\mathrm{SiNx}, 0,70}$ and $\sigma_{A g, 0,70, g g}^{100}=Y_{A g}^{100} \varepsilon_{A g, 0,70, g g}^{100}$.

From this point, we can now imagine conducting a bulge test to find the stress at the end of the annealing process, $\sigma_{A g, p, 70, g g}^{100}$; that is, the stress in the (100)-oriented Ag film at pressure and 
temperature at the end of the annealing process. We simply apply pressure $p$ and find $h$ from the bulge equation, which now has the form

$$
p=\frac{2}{a^{2}}\left(\sigma_{S i N x, 0,70} t_{S i N x}+\sigma_{A g, 0,70, g g}^{100} t_{A g}\right) h+\frac{4}{3 a^{4}}\left(S_{S i N x} t_{S i N x}+S_{A g}^{100} t_{A g}\right) h^{3},
$$

and find the stress in the bulged film from

$$
\sigma_{A g, p, 70, g g}^{100}=Y_{A g}^{100} \varepsilon_{A g, 0,70, g g}^{100}+S_{A g}^{100} \frac{2 h^{2}}{3 a^{2}} .
$$

The resulting values of $\sigma_{A g, p, 70, g g}^{100}$ are shown in Table 1 . The values of the bulge strain are $0,3.23$ $\times 10^{-4}$, and $1.08 \times 10^{-3}$ for the films bulged using $p=0,5$, and $13 \mathrm{kPa}$, respectively (also shown in Table 1).

To find the stress in the (111)-oriented film at the beginning of the annealing process, $\sigma_{A g, p, 70}^{111}$ , we first imagine releasing the bulge pressure and returning to $\sigma_{A g, 0,70, g g}^{100}$. By removing the bulge pressure, we take advantage of the fact that the hypothetical bilayer membrane remains flat during subsequent calculations, so we may assume that the strain in the nitride layer does not change. Thus, we can calculate the strains in the Ag due to grain growth and the change in stress due to texture transformation without having to calculate a force balance between $\mathrm{Ag}$ and $\mathrm{SiN}_{\mathrm{x}}$ at each step, as would be the case if the Ag film in the bilayer transformed under pressure. As before, it does not matter which order we choose. In this case we first imagine shrinking the grains back to their as-deposited size. The residual strain in the Ag layer is then

$$
\varepsilon_{A g, 0,70}^{100}=\varepsilon_{A g, 0,70, g g}^{100}+\delta\left(\frac{1}{d_{\text {asdep }}}-\frac{1}{d_{\text {annealed }}}\right),
$$

where $\delta$ is the thickness of the grain boundary (excess volume per unit area) and $d_{\text {asdep }}$ and $d_{\text {annealed }}$ are the grain sizes of the as deposited and annealed films, respectively. Based on measurements of blanket films prepared in the same equipment under nominally identical conditions, our films are expected to have initial grain sizes of about $90 \mathrm{~nm}$ [30]. Final grain sizes in similar films annealed at similar temperatures (without added bulge stresses) were about $1800 \mathrm{~nm}$ [31]. Using 
these values and assuming $\delta=145 \mathrm{pm}(b / 2$ [26], where $b$ is the magnitude of the Burgers vector [32]), we find the strain due to shrinking of the grains to be $-1.53 \times 10^{-3}$ (Table 1). Note that this shows that grain growth provides the largest strain in the problem $\left(\Delta \varepsilon_{\mathrm{th}}=-8.28 \times 10^{-4}\right.$ (Eq. 6 , heating), $\Delta \varepsilon_{\mathrm{b}, \max }=1.08 \times 10^{-3}(\mathrm{Eq} .7$, for the film tested at $p=13 \mathrm{kPa})$, and $\Delta \varepsilon_{\mathrm{gg}}=1.53 \times 10^{-3}$ (Eq. 8)).

We then allow the orientation of the Ag layer to change to (111). The film remains flat in this step so the strain does not change $\left(\varepsilon_{A g, 0,70}^{111}=\varepsilon_{A g, 0,70}^{100}\right)$. We again apply Hooke's law, $\sigma_{A g, 0,70}^{111}=Y_{A g}^{111} \varepsilon_{A g, 0,70}^{111}$, using $Y_{A g}^{111}=169 \mathrm{GPa}[17]$ leading to a stress of $\sigma_{A g, 0,70}^{111}=-173 \mathrm{MPa}$. Since we assume that the bilayer remains flat until it is bulged, the changes in orientation and grain size in the Ag film do not change the residual strain in the nitride layer. Thus, the residual stress in the nitride is just $\sigma_{\mathrm{SiNx}, 0,70}=Y_{\mathrm{SiN} x} \varepsilon_{\mathrm{SiN}, 0,70}$.

To find the stress in the (111)-oriented $\mathrm{Ag}$ film at pressure and temperature, we simply apply pressure $p$ and find $h$ from the bulge equation, which now has the form

$$
p=\frac{2}{a^{2}}\left(\sigma_{S i N x, 0,70} t_{S i N x}+\sigma_{A g, 0,70}^{111} t_{A g}\right) h+\frac{4}{3 a^{4}}\left(S_{S i N x} t_{S i N x}+S_{A g}^{111} t_{A g}\right) h^{3},
$$

and find the stress in the bulged film from

$$
\sigma_{A g, p, 70}^{111}=Y_{A g}^{111} \varepsilon_{A g, 0,70}^{111}+S_{A g}^{111} \frac{2 h^{2}}{3 a^{2}} .
$$

The values for the films tested at 5 and $13 \mathrm{kPa}$ are -35 and $31 \mathrm{MPa}$, respectively. Note that though the $\mathrm{Ag}$ film in the sample tested at $5 \mathrm{kPa}$ is in compression, the overall stress in the bilayer membrane (Eq. 2) remains tensile due to the large tensile stress in the nitride layer, so our assumption that the bilayer remains flat is valid. However, for the film tested at $0 \mathrm{kPa}$, this assumption is invalid as the overall stress in the bilayer (Eq. 2) must be compressive as the film is observed to be wrinkled when no pressure is applied. To find the stresses in the wrinkled films we simply determine the stresses in the bilayer if it were held flat at $70^{\circ} \mathrm{C}$ and then allow the bilayer to buckle. Values of $\sigma_{A g, 0,70}^{111}$ and $\sigma_{S i N x, 0,70}$ for a flat membrane can be found using thermal 
expansion coefficients as described above. We then allow the bilayer to buckle by adding an additional strain $\varepsilon_{w}$ to both layers until the overall stress in the bilayer is zero. Equation 2 becomes:

$$
F_{T}=F_{1}+F_{2}=\sigma_{1} t_{1}+\sigma_{2} t_{2}=Y_{S i N x}\left(\varepsilon_{S i N x, 0,70}+\varepsilon_{w}\right) t_{S i N x}+Y_{A g}^{111}\left(\varepsilon_{A g, 0,70}+\varepsilon_{w}\right) t_{A g}=0
$$

We find that $\varepsilon_{w}=4.94 \times 10^{-4}$, and that the initial stress in the (111)-oriented $0 \mathrm{kPa} \mathrm{Ag}$ film at $70^{\circ} \mathrm{C}, \sigma_{A g, p, 70}^{111}$, is $-83 \mathrm{MPa}$. The values found for $\sigma_{A g, p, 70}^{111}$ for all three films are shown in Table 1 and Fig. 5.

\section{DISCUSSION}

Figure 5 shows both that the Ag films transformed nearly completely from (111) to (100) and that the stress applied via the bulge test had no discernable effect on the texture transformation kinetics. When overlaid one atop the other in Fig. 5d, the data from the three samples are indistinguishable to within about $5 \%$. To further quantify this comparison, a modified JohnsonMehl-Avrami-Konavolav (JMAK) equation [17],

$$
f_{100}(t)=c\left[1-\exp \left(-K\left(t-t_{0}\right)^{n}\right)\right]
$$

was fit to the $f_{100} v s$. time data shown in Fig. 5. In this expression, $t$ is the measured time in the experiment and $t_{0}$ is the time at which the texture transformation can be thought to have started (accounts for the partial transformation prior to x-ray measurement of the films tested at $p=5$ and $13 \mathrm{kPa}), K$ is determined by an Arrhenius relation that contains both nucleation and growth, $n$ is the Avrami exponent, and $c \leq 1$ is a constant that allows for a transformation that does not proceed to completion but approaches a stable mixed texture. The fit parameters, $c, K, t_{0}$, and $n$, are reported in Table 2. (It should be noted that due to the missing initial transformation data for the films tested at $5 \mathrm{kPa}$ and at $13 \mathrm{kPa}$, a range of values for these parameters produce fits of reasonable quality. Since the three films were the same in their as-deposited state, we simply set the time at the first measurement point for the 5 and $13 \mathrm{kPa}$ tests to the same time as that of the 
equivalent $f_{100}$ value for the $0 \mathrm{MPa}$ test.) In accord with previous studies [15, 17], the fits show transformation behavior that is consistent with nucleation and growth. The values of $n \approx 3$ suggest 2-D growth [33] as would be expected in a film.

The lack of any significant difference between these curves is remarkable. While it has been demonstrated that films can transform without the level of stress that is required according to the interface/strain energy model $[10,15,17]$, it is surprising that the rather significant differences in stress (and, therefore necessarily strain energy) imposed by the bulge tester had no discernable effect on the transformation. This suggests that the actual driving force is significantly larger than the strain energy driving force.

To understand this, we calculate that portion of the driving force that can be attributed to strain energy changes during the bulge test and compare it to other possible driving forces in the problem. Using the stresses under pressure at the beginning $\left(\sigma_{A g, p, 70}^{111}\right)$ and end $\left(\sigma_{A g, p, 70, g g}^{100}\right)$ of the texture transformation (points marked with stars in Fig. 6), we calculate the difference in the strain energy due to texture transformation in each silver layer from

$$
\Delta \phi_{T T}=\frac{\left(\sigma_{A g, p, 70, g g}^{100}\right)^{2}}{S_{A g}^{100}}-\frac{\left(\sigma_{A g, p, 70}^{111}\right)^{2}}{S_{A g}^{111}},
$$

and come up with another surprising result. We find $\Delta \phi_{\mathrm{TT}}$ to be $-21 \mathrm{~kJ} / \mathrm{m}^{3}, 41 \mathrm{~kJ} / \mathrm{m}^{3}$ and 160 $\mathrm{kJ} / \mathrm{m}^{3}$ for the samples with no applied pressure, $5 \mathrm{kPa}$ and $13 \mathrm{kPa}$ applied pressure, respectively (Table 1) - that is, this analysis predicts that the strain energy actually increases during the transformation for all but the sample tested at $0 \mathrm{kPa}$. This arises because the strain energy is raised more by the increase in the tensile strain due to grain growth (Eq. 13) than it is decreased by the reduction in modulus that accompanies the orientation change. Because the strain due to grain growth is tensile, increases in bulge pressure increasingly oppose the grain growth associated with texture transformation. However, this thermodynamic approach to texture transformation does not require that the strain energy decline due to the transformation, only that the energy be reduced relative to the case in which the orientation does not change. Thus, to 
translate this into a driving force for texture transformation, we calculate the change in strain energy, $\Delta \phi_{\mathrm{GG}}$, for the same grain growth (with bulge pressure at $70^{\circ} \mathrm{C}$ ) but without the orientation change and find $\Delta \phi_{\mathrm{GG}}$ to be $-28,154$, and $357 \mathrm{~kJ} / \mathrm{m}^{3}$, for the samples tested under 0,5 , and 13 $\mathrm{kPa}$ applied bulge pressure, respectively. The differences, $\Delta \phi_{\mathrm{TT}}-\Delta \phi_{\mathrm{GG}}$, give the driving forces for texture transformation as $7,-113$, and $-198 \mathrm{~kJ} / \mathrm{m}^{3}$ for the three increasing bulge pressures. Thus, by using the bulge test to impose initial stresses that spanned nearly $140 \mathrm{MPa}$ including both tension and compression, we were able to vary the driving force for texture transformation by more than $200 \mathrm{~kJ} / \mathrm{m}^{3}$. For comparison, a difference in interface energies of $\Delta \gamma=\gamma_{100}-\gamma_{111}=$ $0.184 \mathrm{~J} / \mathrm{m}^{2}$ [17], in a $1200 \mathrm{~nm}$ film provides an energy change of $153 \mathrm{~kJ} / \mathrm{m}^{3}$. So the interface/strain energy model would then predict that the film tested under $13 \mathrm{kPa}$ bulge pressure should transform, but the others should not. The fact that the transformation kinetics are unaffected regardless of whether the strain energy assists the transformation by decreasing 198 $\mathrm{kJ} / \mathrm{m}^{3}$ (film tested at $13 \mathrm{kPa}$ ) or opposes the transformation by increasing by $7 \mathrm{~kJ} / \mathrm{m}^{3}$ (film tested at $0 \mathrm{kPa}$ ) indicates that the actual driving force for the transformation must be much larger than the difference between these two cases, i.e. much larger than $\approx 200 \mathrm{~kJ} / \mathrm{m}^{3}$. (It is interesting to note that, for the films tested at 5 and $13 \mathrm{kPa}$ bulge pressure, some of the increase in strain energy for the transforming films arises because the film undergoes additional strain under constant stress due to the transformation to a lower modulus orientation, so the bulge pressure does work on the film. Use of the load-controlled bulge test thus slightly lowers the driving force for texture transformation compared with a strain-controlled experiment. For example, this accounts for about $19 \%$ of the calculated strain energy change in the film tested at $13 \mathrm{kPa}$.)

With this requirement in mind, we can now consider elimination of defects in the film during grain growth, and the accompanying reduction in total defect energy, as potential driving forces. Of course, a significant driving force arises from elimination of grain boundary area due to grain growth itself. Assuming a grain boundary energy of $1 \mathrm{~J} / \mathrm{m}^{2}$ and a hexagonal columnar grain structure, the total change in grain boundary energy for growth of grains from 90 to $1800 \mathrm{~nm}$, as considered above, is about $21,100 \mathrm{~kJ} / \mathrm{m}^{3}$. This is two orders of magnitude larger than our 
calculated strain energy driving force, which may explain why the transformation is insensitive to the applied bulge strains. Following the logic of the interface/strain energy model, the energy in a film that changes orientation is only $50 \mathrm{~kJ} / \mathrm{m}^{3}\left(-198 \mathrm{~kJ} / \mathrm{m}^{3}\right.$ strain energy $+153 \mathrm{~kJ} / \mathrm{m}^{3}$ interface energy) lower than that of a film that does not, or $0.2 \%$ of the grain boundary driving force. The actual driving force for the orientation change can't be too much smaller than $21,100 \mathrm{~kJ} / \mathrm{m}^{3}$ to be able to influence the grain growth process.

Accordingly, we calculate the change in energy due to elimination of point, line, and planar defects within the grains - that is, vacancies, dislocations, and twin boundaries - to determine if and when reduction in these defect energies would be capable of influencing orientation selection during grain growth in as-deposited PVD films. This process is a form of recrystallization [34], in which new defect-free grains grow at the expense of pre-existing grains containing high densities of defects. As with the strain energies, it is important to emphasize that the defect energy magnitude alone is not sufficient. An orientation-dependent differential in such energies would be required to generate a texture transformation.

Sonnweber-Ribic et al. [15], studied texture transformations in $\mathrm{Cu}$ films and estimated the potential driving force due to elimination of dislocations by assuming that all of the peak width that they observed in x-ray diffraction arose from dislocations. Their analysis gave a dislocation density of $1.25 \times 10^{14} / \mathrm{m}^{2}$. Reducing this to zero would recover about $170 \mathrm{~kJ} / \mathrm{m}^{3}$, which would just barely be enough to overcome the opposing interface energy in our films. While dislocation densities in heavily deformed FCC metals can reach $10^{15}-10^{16} / \mathrm{m}^{2}$, which would translate to total defect energies of 1360-13,600 kJ/m³ , the actual dislocation densities in as-deposited PVD films are not well known. Studies reporting dislocation densities in this range on the basis of TEM investigations $[35,36]$ and analysis of $\mathrm{x}$-ray peak widths $[37,38]$ in as-deposited FCC films have been published. However, Legros et al. [36] note that the densities they obtained using TEM should be considered as upper limits with typical values nearer to $10^{12} / \mathrm{m}^{2}$, and Kamminga et al . [38] note that dislocation densities obtained by XRD methods are not reliable due to the assumptions (sets of infinite, straight, parallel dislocations) underlying the analysis. Thus, while 
reduction in dislocation energy can provide larger driving force magnitudes, very high dislocation densities, in the $10^{15}-10^{16} / \mathrm{m}^{2}$ range, would be required. The extent to which such densities actually exist in as-deposited PVD films remains to be determined. More importantly, the orientation selection mechanism is not clear. Sonnweber-Ribic et al. [15] claimed that the initial dislocation density was higher in (100) grains, which led to more rapid recovery and ultimately to lower dislocation densities in those grains; which, in turn allowed them to grow at the expense of the (111) grains. However, neither the differential in initial dislocation densities, nor the ability of the dislocation density in (100) to recover faster and farther than that in the (111) grains, nor the reason why the (100) grains should then grow were explained. If the interface/strain energy balance adds only $50 \mathrm{~kJ} / \mathrm{m}^{3}$ as we show above, then one might expect defect-free (111) grains to grow at the expense of both (100) and (111) grains containing dislocations in order to minimize both interface and defect energies.

While vacancy densities in PVD (particularly sputtered) films are often said to be high [39], estimating the change in energy due to elimination of vacancies is difficult, as the actual vacancy concentrations in as-deposited metal films seem also not to be well known. However, assuming a vacancy formation energy of $1.85 \times 10^{-19} \mathrm{~J}$ [40], we find that the vacancy concentration must be greater than $3.85 \times 10^{-4}$ in order for the energy change due to vacancy annihilation to exceed the opposing strain energy of the film with the highest applied bulge pressure. For reference, this is higher than the equilibrium vacancy concentration in silver near the melting point $\left(1.6 \times 10^{-4}\right.$ [41]). While such high vacancy densities have been seen in simulations [35] of PVD film deposition, it is not yet clear if they exist in real evaporated Ag films. Thus, as with dislocations, we can conclude that reduction in vacancy energy can provide sufficient driving force to explain our results, but very high vacancy densities, $10^{-3}$ and larger, would be required. Even if this were the case, the same problems with orientation selection as described regarding dislocations above, would exist.

We note that twin boundaries may also play a role in driving the transformation. Thin FCC metal films produced by sputter deposition have been shown to contain very high densities of 
twin boundaries in their as-deposited state $[4,6,14,42]$, including several where texture transformation subsequently occurred $[4,6,14]$. Nanotwins with twin boundary spacings of order $10 \mathrm{~nm}$ have been reported in Ag films prepared by magnetron sputtering [42]. Assuming a twin boundary energy of $0.01 \mathrm{~J} / \mathrm{m}^{2}$ and a twin spacing of $10 \mathrm{~nm}$, a driving force of $1000 \mathrm{~kJ} / \mathrm{m}^{3}$ could be realized if these defects were eliminated by the texture transformation. While still much smaller than the driving force due to elimination of grain boundaries, this value meets the criterion of significantly exceeding the strain and interface energies in our films. Furthermore, it is possible for nanotwins to provide an orientation selection mechanism. For example, if only (111) grains show such densities of nanotwins [39], then those grains would not grow, while nanotwin-free grains would. Why (100) grains should be singled out from among all other orientations for growth remains to be determined.

Thus, we conclude that, while stored defect energy in the form of dislocations and vacancies could, in principle, provide energies that are large compared with the (obviously ineffective) interface and strain energy contributions and thus might drive the observed texture transformation, very high densities of these defects would be required, and the orientation selection mechanism is not obvious. In contrast, a recrystallization process driven by elimination of excess energy in the form of twin boundaries in experimentally reported densities could provide that driving force, and a simple orientation selection mechanism may be possible.

It is important to note that the approach we are taking here, like the canonical interface/strain energy model [2-4], is purely thermodynamic. This approach runs into conceptual difficulties when we consider that the driving force for grain growth is as much as 20 times greater than the other defect-energy-driven processes we considered as candidates that might lead to texture transformation. If we accept a simple picture in which the grain boundary velocity scales with the product of the driving force and the grain boundary mobility, neglect any differences in the grain boundary mobility, and assume that (100) grains - which are not evident in the initial structures in many films that undergo texture transformation $[7,10,11,12,14,16,17]-$ nonetheless exist or are rapidly formed during the initial stages of the transformation, then we 
would at best expect a $5 \%$ differential in driving force to result in only a small difference in the final texture. That is, both the existing (111) grains and any existing (100) grains should grow, but the (100) grains should grow only slightly faster such that the final texture would contain only slightly more (100) than would have been the case without the differential driving force. The fact that in many cases a virtually $100 \%$ (111) texture is replaced with a virtually $100 \%$ (100) texture $[7,10,11,14,16,17]$ suggests that a kinetic argument might be more appropriate, or at least a case where grain boundary energies were highly anisotropic. For example, situations where the energies or mobilities of $(111) /(111)$ boundaries were significantly lower than those of $(111) /(100)$ boundaries [12], or where the only new defect-free nuclei created had the (100) orientation, would be much more consistent with the experimental data.

As described in Section 2, the $\mathrm{Ag} / \mathrm{Si}_{3} \mathrm{~N}_{4}$ bilayers were wrinkled in the as-deposited state indicating that they were in net compression. Due to the wrinkling, the stresses in the asdeposited membranes could not be directly measured. Additional experimental constraints prevented us from being able to determine the stresses in-situ during the transformation. As a result, a circuitous route was followed to estimate the stresses (Fig. 6), and thus the strain energy available to drive the texture transformation $(\mathrm{Eq} 12)$. We can obtain a rough check on the validity and accuracy of those stresses as follows: Since the as-deposited films were wrinkled, the $\mathrm{Ag} / \mathrm{Si}_{3} \mathrm{~N}_{4}$ bilayers were in net compression, and since we know the stress in the nitride layer was tensile at $424 \mathrm{MPa}$, we can use Eq. 3 to find that the residual stress in the as-deposited silver films at room temperature, $\sigma_{A g, 0, R T}^{111}$, must be more compressive than -76 MPa. By comparison, if we add the initial thermal strain (Eq. 6) to take our calculated value of $\sigma_{A g, 0,70}^{111}$ back to $\sigma_{A g, 0, R T}^{111}$, we find a value of $-33 \mathrm{MPa}$. This is actually quite good agreement considering the assumptions that were made during the calculations, suggesting that the stresses found for $\sigma_{A g, 0,70}^{111}$ and $\sigma_{A g, 0, R T}^{111}$ are reasonable. For example, we assumed a grain boundary width of $\delta=b / 2$ in Eq. 13, a fairly common assumption [26]. An increase of only $15 \%$ in $\delta$, or a decrease of only $13 \%$ in the initial grain size (taken from TEM measurements [30]) would be sufficient to result in a value for $\sigma_{A g, 0,70}^{111}$ of $-76 \mathrm{MPa}$. Similarly, an error in the measurement of the residual stress in the Ag layer 
at the end of the experiment of only $17 \mathrm{MPa}$ would meet this requirement. Finally, we ignored plasticity in our calculations for two reasons. First, in thermal cycles of very similar films (same deposition process, Ti interlayer, and thickness), significant plastic deformation was not observed until temperatures were above $100^{\circ} \mathrm{C}$ and stresses above $100 \mathrm{MPa}$ [16]. Second, our calculations resulted in very similar predicted stresses for the films in the as-deposited state for all three samples. Had different amounts of plastic strain occurred in the films due to the different applied bulge pressures, we would have ended up predicting different stresses in the asdeposited films. This would have meant that three nominally identical films prepared in the same way would have had significantly different initial stresses. As this is unlikely, it must be the case either that plastic deformation is small or that it is the same in all films, despite rather different loadings. A crude estimate based on allowing both tensile and compressive stresses to relax in the same way suggests that the calculated stresses are probably within several tens of MPa of the actual values, which, in turn, suggests that the strain energies are known within about $100 \mathrm{~kJ} / \mathrm{m}^{3}$. Our main conclusions are not affected by variations in stresses at this level.

\section{SUMMARY AND CONCLUSIONS}

The established model that describes the thickness-dependent (111) to (100) texture transformation in thin FCC metal films in terms of a competition between strain and interface energies [2-4] has been challenged by reports in which films transformed although the required strain energy was not present $[10,11,15-17]$. A recrystallization process driven by reduction in dislocation energy as an alternative driving force has been proposed [15]. On the assumption that many different driving forces might contribute to the texture transformation, we investigated those driving forces in the current work by using a bulge test apparatus to impose different strain states to otherwise nominally identical thin Ag films during annealing to vary the strain energy driving force as much as possible. The corresponding texture transformation behavior was characterized using in-situ synchrotron x-ray diffraction. Since stresses could not be measured in-situ, the residual stress in the films after annealing was determined using the bulge test and the 
stresses at the beginning and end of the transformation were determined using an analysis that included strains due to differential thermal expansion, applied bulge strains, and strains that arise due to elimination of excess grain boundary volume during grain growth.

Strains due to grain growth have not generally been considered in reports of the (111)-to(100) texture transformation (for example, Thompson and Carel specifically assumed its effects would be negligible [47]), but our analysis makes it clear that they are too large to be ignored. The strain due to grain growth $\left(1.53 \times 10^{-3}\right)$ is much larger than either the thermal strain $(-8.28 \times$ $\left.10^{-4}\right)$ or the largest applied bulge strain $\left(1.08 \times 10^{-3}\right)$.

For a certain amount of grain growth, the difference in energy between a film that changes orientation and one that does not gives the driving force for texture transformation. Application of different bulge strains successfully varied the strain energy contribution from $+7 \mathrm{~kJ} / \mathrm{m}^{3}$ to -198 $\mathrm{kJ} / \mathrm{m}^{3}$. Considering an opposing change in interface energy of $153 \mathrm{~kJ} / \mathrm{m}^{3}$ [17] these results should have produced films with and without texture transformation according to the widely accepted interface/strain energy model [2-4]. However, despite these significant differences in strain energy, the kinetics of the (111)-to-(100) transformation were unaffected, indicating that the actual driving force for the transformation is much larger.

Assuming that the texture-transformation is a recrystallization process, driven by the reduction in energy that occurs when new defect-free grains grow to replace existing defectcontaining grains, we calculated the defect densities that would be necessary to meet this criterion if those defects were dislocations, vacancies, or twin boundaries. Dislocation and vacancy densities would have to exist at very high levels and the orientation selection mechanism is not obvious. However, the necessary driving force can be provided by twin boundary densities similar to those reported experimentally in nanotwinned Ag films prepared by magnetron sputtering $[42]\left(\approx 1000 \mathrm{~kJ} / \mathrm{m}^{3}\right)$, and an orientation selection mechanism is possible. Additional work to directly confirm the correlation between the presence of the necessary twin boundary density and their elimination during texture transformation is required. 
We note that, even in the most extreme cases, the additional driving forces considered are only a fraction of the driving force available for grain growth, suggesting that a simple driving force model may not be appropriate to describe the texture transformation process.

\section{ACKNOWLEDGMENTS}

This work was supported by the National Science Foundation (NSF) via award DMR-1106223. This work is based upon research partially conducted at the Cornell High Energy Synchrotron Source (CHESS) which is supported by NSF and the National Institutes of Health/National Institute of General Medical Sciences under NSF award DMR-0936384. M-T L received support for a sabbatical visit at Cornell University from the Taiwan National Science Council under grant No. NSC 101-2918-I-005-005. The authors would like to thank Prof. Michael O. Thompson for assistance with the construction and operation of the interferometer. 


\section{REFERENCES}

1. Polop C, Rosiepen C, Bleikamp S, Drese R, Mayer J, Dimyati A, Michely T, The STM view of the initial stages of polycrystalline Ag film formation, New J Phys 2007;9.

2. Floro JA, Thompson CV, Carel R, Bristowe PD, Competition between strain and interface energy during epitaxial grain growth in Ag films on Ni(001), J Mater Res 1994;9:2411,

3. Zielinski EM, Vinci RP, Bravman JC, Effects of barrier layer and annealing on abnormal grain growth in copper thin films, J Appl Phys 1994;76:4516.

4. Vook, RW, Witt F, Structure and annealing behavior of metal films deposited on substrates near 80K: I. Copper films on glass, J Vac Sci Technol 1965;2:49.

5. Patten JW, McClanahan ED, Johnston JW, Room-temperature recrystallization in thick biassputtered copper deposits, J Apply Phys 1971;42:4371.

6. Dahlgren SD, Columnar grains and twins in high-purity sputter-deposited copper, J Vac Sci Technol 1974;11:832.

7. Ohmi T, Saito T, Otsuki M, Shibata T, Nitta T, Formation of copper thin films by a low kinetic energy particle process, J Electrochem Soc 1991;138:1089.

8. Zielinski EM, Vinci RP, Bravman JC, The influence of strain energy on abnormal grain growth in copper thin films, Appl Phys Lett 1995;67:1078.

9. Zielinski EM, Vinci RP, Bravman JC, The effects of processing on the microstructure of copper thin films on tantalum barrier layers, MRS Online Proc Libr 1995;391.

10. Greiser J, Muller D, Mullner P, Thompson CV, Arzt E, Growth of giant grains in silver thin films, Scripta Mater 1999;41:709.

11. Greiser J, Mullner P, Atzt E, Abnormal growth of giant grains in silver thin films, Acta Mater 2001;49:1041.

12. Koike J, Wada M, Sanada M, Maruyama K, Effects of crystallographic texture on stressmigration resistance in copper thin films, Appl Phys Lett 2002;81:1017.

13. Sonnweber-Ribic P, Gruber P, Dehm G, Arzt E, Texture transition in copper thin films: Electron backscatter diffraction vs. x-ray diffraction, Acta Mater 2006;54:3863.

14. Vanstreels K, Brongersma SH, Tokei Z, Carbonell L, De Ceuninck W, D’Haen J, D'Olieslaeger M, Increasing the mean grain size in copper films and features, J Mater Res 2008;23:642.

15. Sonnweber-Ribic P, Gruber PA, Dehm G, Strunk HP, Arzt E, Kinetics and driving forces of abnormal grain growth in thin $\mathrm{Cu}$ films, Acta Mater 2012;60:2397.

16. Baker SP, Saha K, Shu JB, Effect of thickness and Ti interlayers on stresses and texture transformation in thin Ag films during thermal cycling, Appl Phys Lett 2013;103:191905.

17. Baker SP, Hoffman B, Timian L, Silvernail A, Ellis EA, Texture transformations in Ag thin films, Acta Mater 2013;61:7121.

18. Thompson CV, Carel R, Texture development in polycrystalline thin films, Mater Sci Eng B 1995;32:211.

19. Thompson CV, Texture evolution during processing of polycrystalline films, Ann Rev Mater Sci 2000;30:159. 
20. Nowak DE, Blasini DR, Vodnick AM, Blank B, Tate, MW, Six-circle diffractometer with atmosphere-and temperature-controlled sample stage and area and line detectors for use in the G2 experimental station at CHESS, Rev Sci Instrum 2006;77:113301.

21. Mongkolsuttirat, Kittisun, Time and Temperature Dependence of Viscoelastic Stress Relaxation in Gold and Gold Alloy Thin Films, PhD Dissertation, Lehigh University, 2013.

22. Vlassak JJ, Nix WD, A new bulge test technique for the determination of Young's modulus and Poisson's ratio of thin films, J Mater Res 1992;7:3242.

23. Xiang Y, Chen X, Vlassak JJ, Plane-strain bulge test for thin films, J Mater Res 2005;20:2360.

24. Neighbors JR, Alers GA, Elastic constants of silver and gold, Phys Rev 1958;111:707.

25. Small MK, Nix WD, Analysis of the accuracy of the bulge test in determining the mechanical properties of thin films, J Mater Res 1992;7:1553.

26. Chaudhari, P, Grain-growth and stress relief in thin-films. J Vac Sci Technol 1972;9(1):520.

27. Fransila, Sami, Introduction to Microfabrication, $2^{\text {nd }}$ Ed., John Wiley \& Sons, Chichester, West Sussex, England, 2010.

28. Hughey MP, Cook RF, Massive stress changes in plasma-enhanced chemical vapor deposited Silicon nitride films on thermal cycling, Thin Solid Films 2004;460:7.

29. Ashby, Michael F, Materials and the Environment, Butterworth-Heinemann, 2012.

30. Baker SP, Ellis EA. In progress.

31. Dorokhov S, Visser K, Baker SP. In progress.

32. Dubiel M, Brunsch S, Troger L, Temperature dependence of thermal expansion coefficient of silver nanoparticles and bulk material determined by EXAFS, J Synch Rad 2001;8:539.

33. Avrami MJ, Kinetics of phase change, I: General theory, Chem Phys 1939;7:1103.

34. Humphreys, F. J. and M. Hatherly, Recrystallization and related annealing phenomena, $2^{\text {nd }}$ Ed., Elsevier, 2004.

35. Amin-Ahmadi B, Idrissi H, Galceran M, Colla MS, Raskin JP, Pardoen T, Godet S, Schryvers D, Effect of deposition rate on the microstructure of electron beam evaporated nanocrystalline palladium thin films, Thin Solid Films 2013;539:145.

36. Legros M, Cabie M, Gianola D, In Situ Deformation of Thin Films on Substrates, Microsc Res Tech 2009;79:270.

37. Csiszar G, Balogh L, Misra A, Zhang X, Ungar T, The dislocation density and twinboundary frequency determined by X-ray peak profile analysis in cold rolled magnetronsputter deposited nanotwinned copper, J of Appl Phys 2011;110:043502.

38. Kamminga JD, Seijbel LJ, Diffraction Line Broadening Analysis if Broadening Is Caused by Both Dislocations and Limited Crystallite Size, J Res Nat Inst Stand Technol;2004;109(1):65.

39. Zhou XW, Johnson RA, Wadley HNG, Vacancy formation during vapor deposition, Acta Mater 1997;45(11):4441.

40. Triftshauser W, McGervey JD, Monovacancy formation energy in copper, silver, and gold by positron annihilation, Appl Phys 1975;6:177.

41. Simmons RO, Balluffi RW, Measurement of the equilibrium concentration of lattice vacancies near the melting point, Phys Rev 1960;119:600. 
42. Bufford D, Wang H, Zhang X, High-strength, epitaxial nanotwinned Ag films, Acta Mater 2011;59: 93.

43. Barmak K, Eggeling E, Kinderlehrer D, Sharp R, Ta'asan S, Rollett AD, Coffey KR, Grain growth and the puzzle of its stagnation in thin films: the curious tale of a tail and an ear, Prog Mater Sci 2013;58(7):987.

44. Mullins WW, Two-dimensional motion of idealized grain boundaries, J Appl Phys;27(8):900.

45. Frost HJ, Thompson C, Walton DT, Simulation of thin film grain structures-I. Grain growth stagnation, Acta Metall Mater;38(8):1455.

46. Gottstein G, Schvindlerman L, Triple Junction drag and grain growth in 2D polycrystals, Acta Mater;50(4):703.

47. Thompson C, Carel R, Stress and grain growth in thin films, J Mech Phys Solids 1996;44(5):657.

\section{FIGURE AND TABLE CAPTIONS}

Figure 1: Schematic of bulge sample and test. (a) View of Si die from below showing $\mathrm{Si}_{3} \mathrm{~N}_{4}$ membrane. (b) Cross section showing sample mounted on bulge tester with $\mathrm{Si}_{3} \mathrm{~N}_{4} / \mathrm{Ti} / \mathrm{Ag}$ membrane bulged up during testing. (c) Schematic cross section of the bulge test. Bulge width $2 a$, height $h$, radius $R$, and pressure $p$ are as illustrated.

Figure 2: Schematic of the x-ray experiment. A 2-D detector was used to capture diffracted intensity from a segment of a diffraction cone. Rocking curves were conducted by rotating about $\omega$.

Figure 3: Detector images showing segments of (a) (222) diffraction ring before annealing, and (b) (400) diffraction ring after annealing. The smooth (222) ring is consistent with the presence of many small (111) grains before annealing and the spotty (400) ring is consistent with the presence of few large (100) grains formed by anomalous grain growth during annealing. (Note that the maximum intensity in each image is shown as white, so brightness does not indicate relative intensities from image to image.)

Figure 4: $\omega$ rocking curves taken during annealing from the sample tested at zero applied bulge pressure. (a) and (b) show, respectively, (222) intensity before annealing and (400) intensity after annealing. (c) and (d) show the evolution of rocking curve intensity 
with time for the (222) and (400) peaks, respectively. The fine-grained (111) texture component is replaced with a coarse-grained (100) texture component in a classic texture transformation.

Figure 5: Volume fractions of the (100) texture component as a function of time during annealing of samples tested under (a) $0 \mathrm{kPa}$, (b) $5 \mathrm{kPa}$, and (c) $13 \mathrm{kPa}$ bulge pressure. (d) Data from all three tests plotted together. X-ray data shown by black symbols; modified Avrami fits (Eq 4) shown by dashed lines. All transformation curves are equal to within $5 \%$.

Figure 6: Schematic showing how the stresses in the silver layers vary with the different steps in the experimental procedure (solid lines) as well as the thought experiment (dashed lines) leading to an estimate of the stress in the (111) Ag films at temperature and under bulge pressure, $\sigma_{A g, p, 70}^{111}$, at the beginning of the texture transformation and of the stress in the (100) Ag films at temperature and pressure, $\sigma_{A g, p, 70, g g}^{100}$, after the texture transformation (these values are indicated with stars). Remaining symbols are defined in the text.

Table 1: Sample and test parameters. Pressure is the applied bulge pressure; membrane size is the dimension of the bulge membrane; $t_{A g}$ is the thickness of the silver layer, $\sigma_{p, 70}^{111}$ and $\sigma_{p, 70, g g}^{100}$ are the stresses in the silver layer before and after the transformation; $R^{2}$ is the correlation coefficient of the Avrami fit; $\Delta \varepsilon_{t h, A g}, \Delta \varepsilon_{b}$, and $\Delta \varepsilon_{g g}$ are the thermal, bulge, and grain growth strains; and $\Delta \phi_{\mathrm{TT}}-\Delta \phi_{\mathrm{GG}}$ is the strain energy driving force for the transformation.

Table 2: Fit parameters for Johnson-Mehl-Avrami-Konavolav analysis. 
Figure 1a

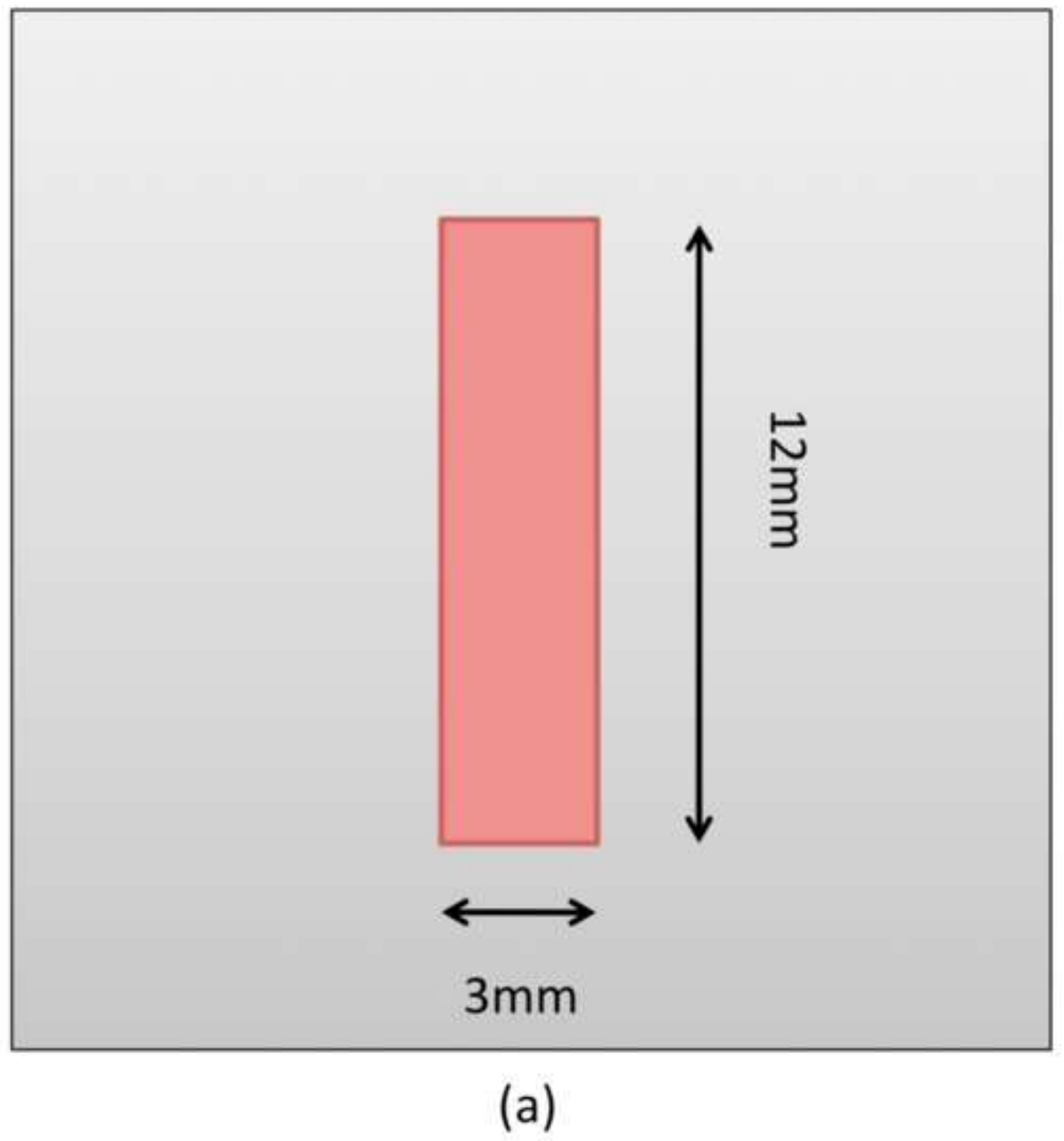

(a) 
(c)

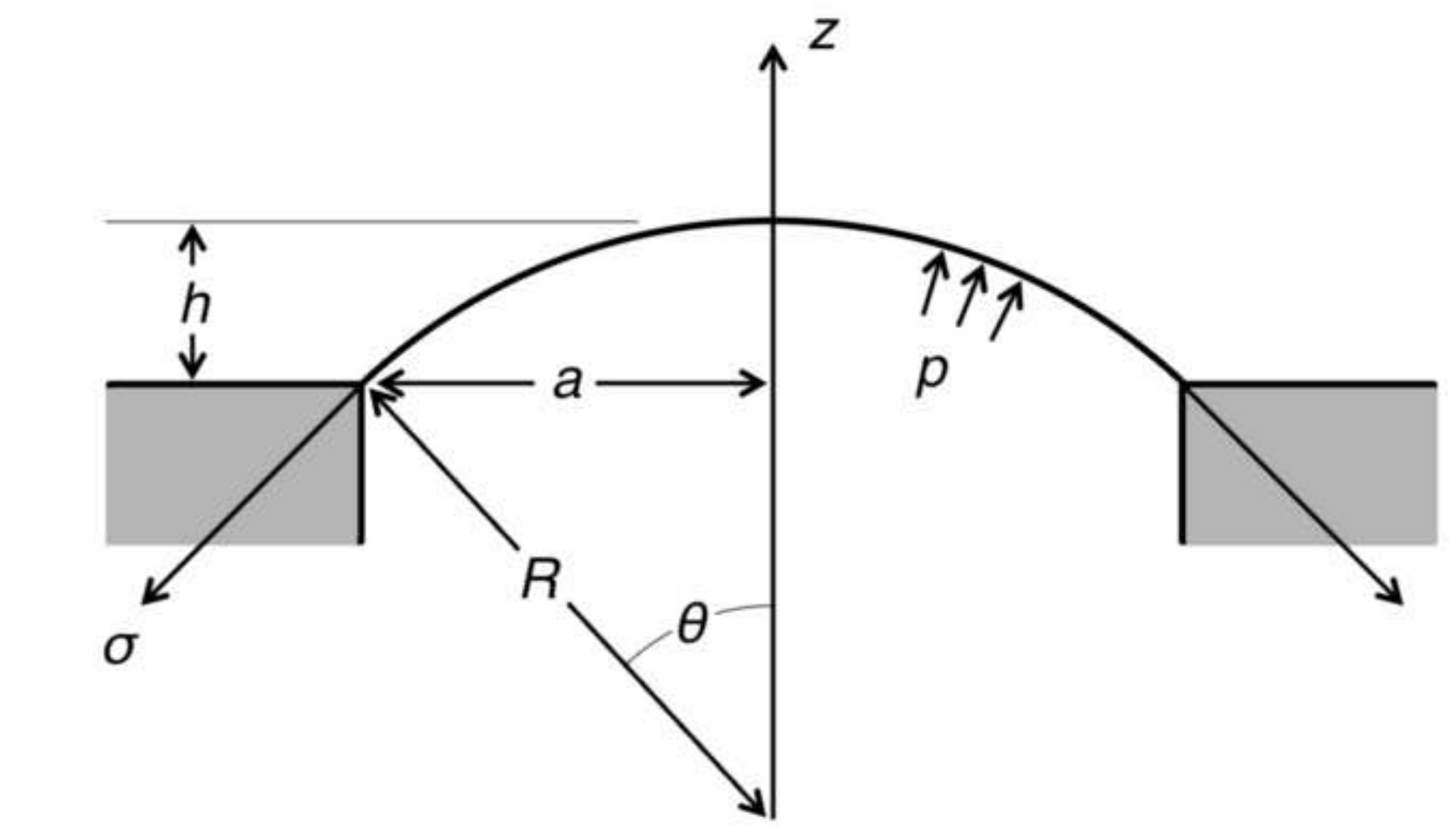

gure$$
\text { . }
$$ 


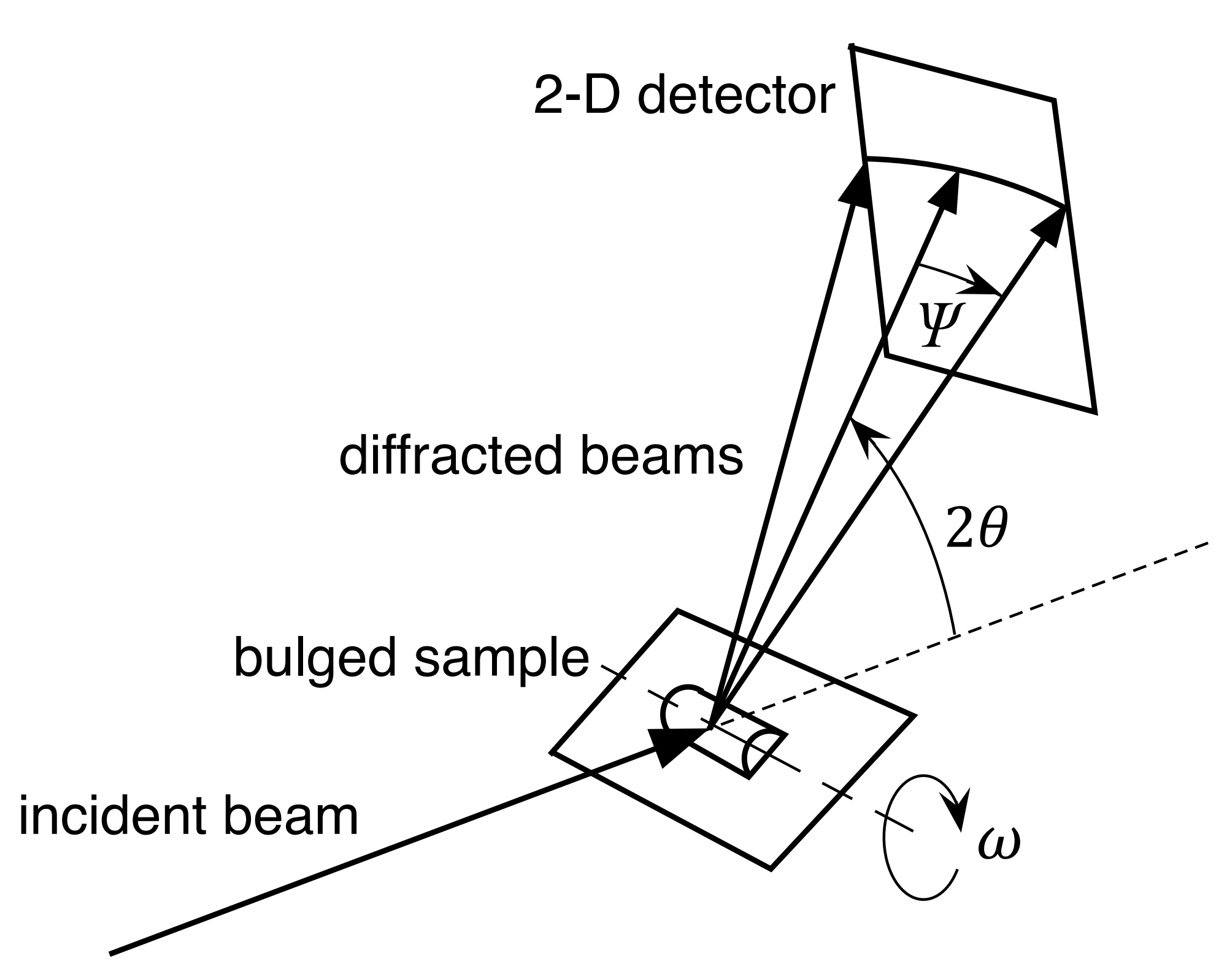

incident beam

Figure 2

(

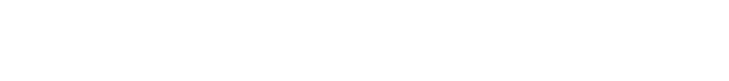

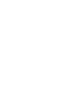

. 


\section{a: (222)}

b: (400)
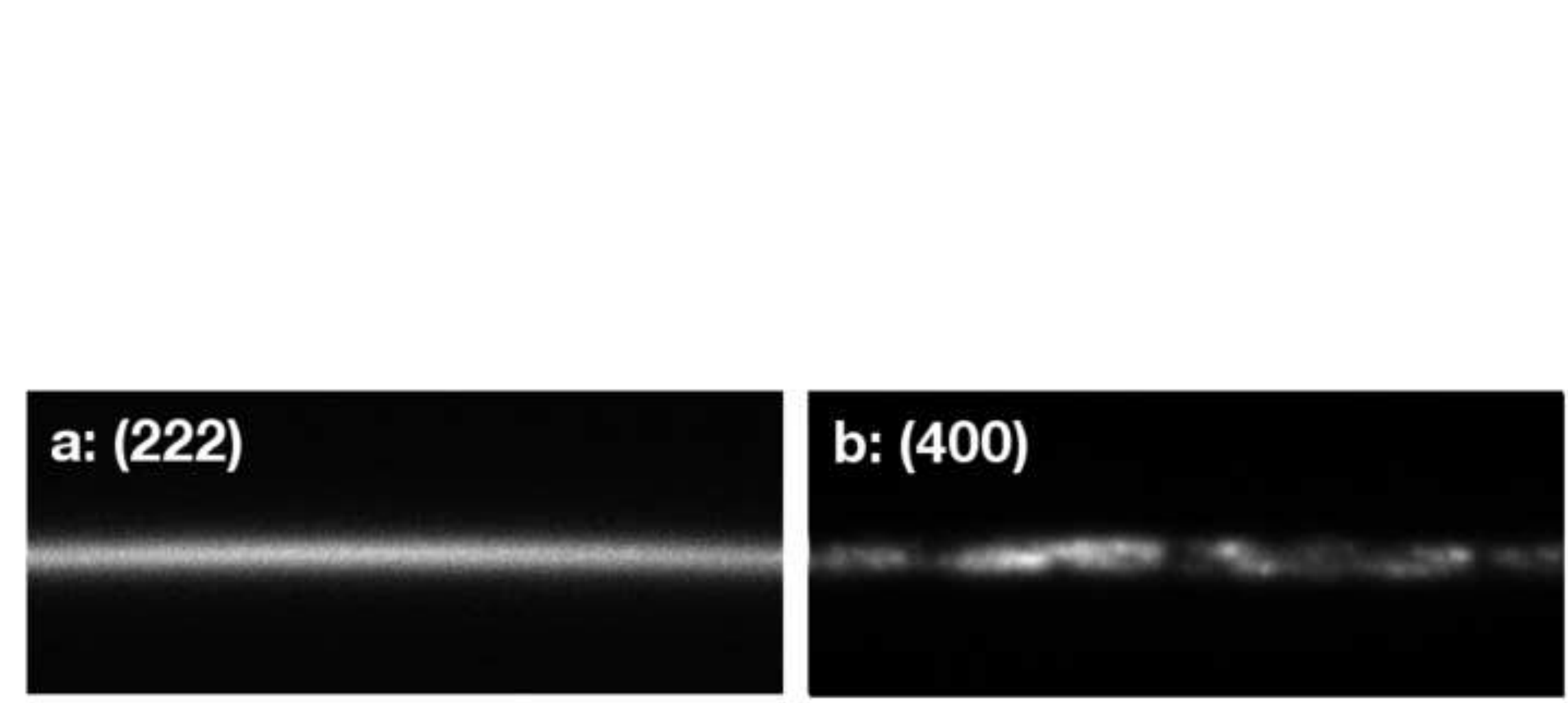
.

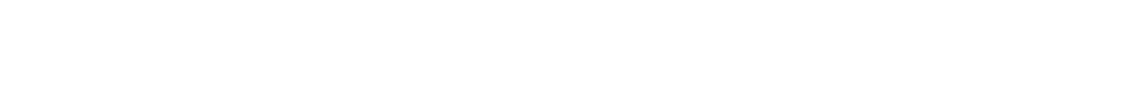




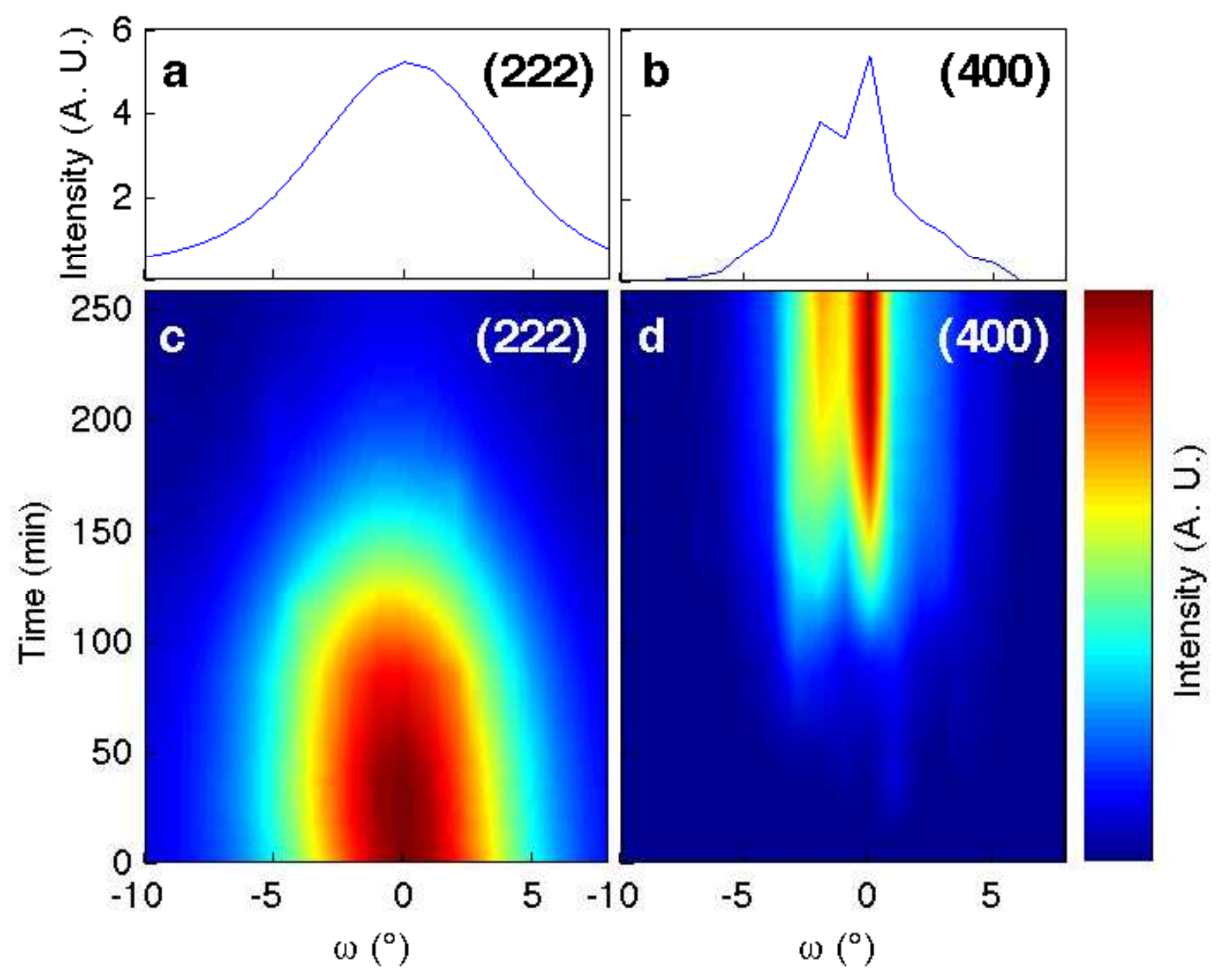

3

$\leq$

蛋

50

0

$-10$

$$
-5
$$

$\omega\left(^{\circ}\right)$

$\omega\left(^{\circ}\right)$ 


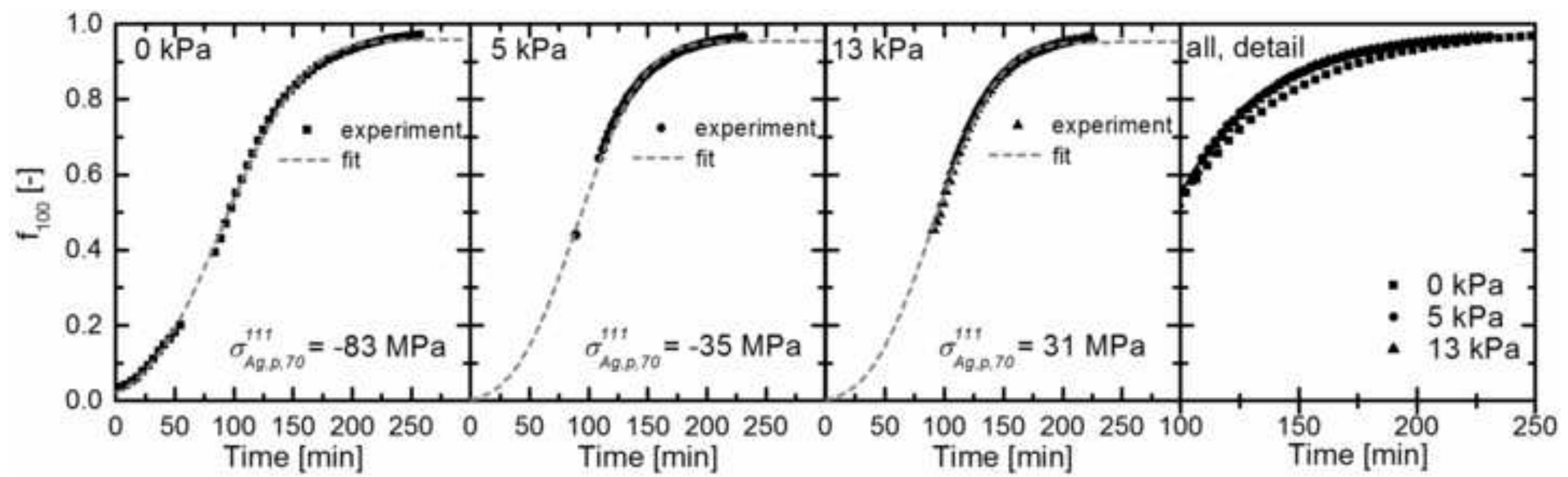




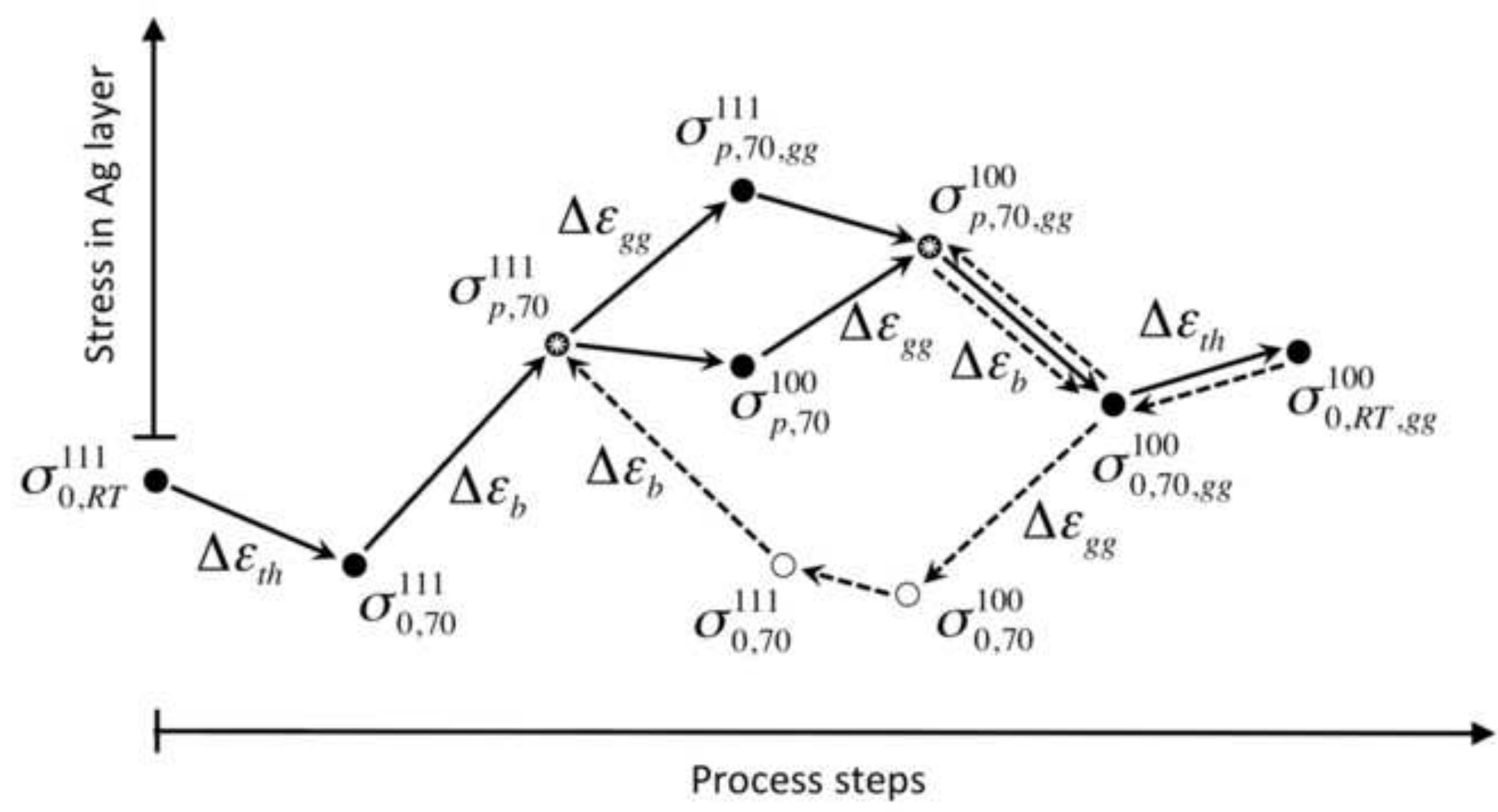

F

Process steps 
Table 1

\begin{tabular}{|c|c|c|c|c|c|c|c|c|c|}
\hline $\begin{array}{c}\text { Pressure } \\
(\mathrm{kPa})\end{array}$ & $\begin{array}{c}\text { Membrane size } \\
(\mathrm{mm} \times \mathrm{mm})\end{array}$ & $\begin{array}{c}t_{\mathrm{Ag}} \\
(\mathrm{nm})\end{array}$ & $\begin{array}{c}\mathrm{T}_{\text {average }} \\
\left({ }^{\circ} \mathrm{C}\right)\end{array}$ & $\begin{array}{c}\sigma_{p, 70}^{111} \\
(\mathrm{MPa})\end{array}$ & $\begin{array}{c}\sigma_{p, 70, g g}^{100} \\
(\mathrm{MPa})\end{array}$ & $\Delta \varepsilon_{t h, A g}$ & $\Delta \varepsilon_{b}$ & $\Delta \varepsilon_{g g}$ & $\begin{array}{c}\Delta \phi_{\mathrm{TT}}-\Delta \phi_{\mathrm{GG}} \\
\left(\mathrm{kJ} / \mathrm{m}^{3}\right)\end{array}$ \\
\hline 0 & $2 \times 12$ & 1204 & 67.8 & -83 & 38 & $-8.28 \times 10^{-4}$ & 0 & $1.53 \times 10^{-3}$ & 7 \\
\hline 5 & $3 \times 12$ & 1179 & 68.2 & -35 & 60 & $-8.28 \times 10^{-4}$ & $3.23 \times 10^{-4}$ & $1.53 \times 10^{-3}$ & -113 \\
\hline 13 & $3 \times 12$ & 1179 & 69.7 & 31 & 108 & $-8.28 \times 10^{-4}$ & $1.08 \times 10^{-3}$ & $1.53 \times 10^{-3}$ & -198 \\
\hline
\end{tabular}


Table 1

\begin{tabular}{|c|c|c|c|c|c|}
\hline $\begin{array}{c}\text { Pressure } \\
(\mathrm{kPa})\end{array}$ & $c$ & $\begin{array}{c}K \\
(1 / \mathrm{min})\end{array}$ & $n$ & $\begin{array}{c}t_{0} \\
(\mathrm{~min})\end{array}$ & $R^{2}$ \\
\hline 0 & 0.9447 & $2.648 \times 10^{-7}$ & 3.012 & -39.15 & 0.9992 \\
\hline 5 & 0.9282 & $1.412 \times 10^{-7}$ & 3.183 & -33.28 & 0.9937 \\
\hline 13 & 0.9098 & $1.064 \times 10^{-7}$ & 3.278 & -25.53 & 0.9965 \\
\hline
\end{tabular}




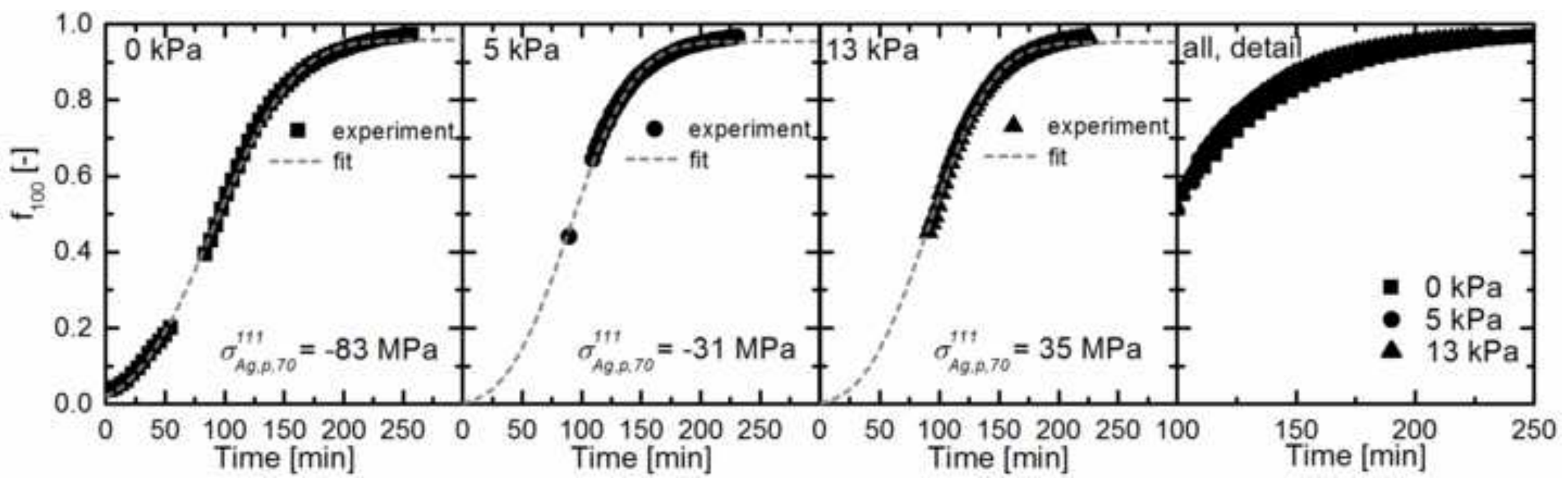

